\title{
Redox control of thermopower and figure of merit in phase-coherent molecular wires
}

\author{
Víctor M. García-Suárez ${ }^{\mathrm{a}, \mathrm{b}}$, Colin J. Lambert ${ }^{\mathrm{a} *}$, David Manrique ${ }^{\mathrm{a}}$ and Thomas \\ Wandlowskic \\ a Department of Physics, Lancaster University, Lancaster LA1 4YB, United Kingdom, \\ c.lambert@lancaster.ac.uk \\ ${ }^{\mathrm{b}}$ Departamento de Física, Universidad de Oviedo and CINN (CSIC), ES-33007 Oviedo, Spain \\ ${ }^{c}$ Department of Chemistry and Biochemistry, University of Bern, Freiestrasse 3, 3012 Bern
}

KEYWORDS:OPE, molecular wires, single molecule conductance, thermopower, STM break junction (STM-BJ), mechanically controlled break junction (MCBJ), ab-inito transport calculations, density functional theory, non-equilibrium Green's functions, quantum interference.

\section{ABSTRACT:}

We demonstrate how redox control of intra-molecular quantum interference in phase-coherent molecular wires can be used to enhance the thermopower (Seebeck coefficient) S and thermoelectric figure of merit ZT of single molecules attached to nanogap electrodes. Using first principles theory, we study the thermoelectric properties of a family of nine molecules, which consist of dithiol-terminated oligo(phenylene-ethynylenes) (OPEs) containing various central units. Uniquely, one molecule of this family possesses a conjugated acene-based central backbone attached via triple bonds to terminal sulfur atoms bound to gold electrodes and incorporates a dihydroanthracene central unit. We demonstrate that both $\mathrm{S}$ and the electronic contribution $\mathrm{Z}_{\mathrm{el}} \mathrm{T}$ to the figure of merit $\mathrm{ZT}$ can be dramatically enhanced by oxidizing the dihydroanthracene to yield a second molecule, which possesses a crossconjugated anthraquinone central unit. This enhancement originates from the conversion of broken pi-conjugation in the former to cross-conjugation in the latter, which promotes the appearance of a sharp anti-resonance at the Fermi energy. Comparison with thermoelectric properties of the remaining seven conjugated molecules demonstrates that such large values of $\mathrm{S}$ and $\mathrm{Z}_{\mathrm{el}} \mathrm{T}$ are unprecedented. We also evaluate the phonon contribution to the thermal conductance, which allows us to compute the full figure of merit $Z T=\mathrm{Z}_{\mathrm{el}} \mathrm{T}\left(1+\kappa_{p} / \kappa_{e}\right)$, where is $\kappa_{p}$ the phonon contribution to the thermal conductance and $\kappa_{e}$ is the electronic contribution. For unstructured gold electrodes, $\kappa_{p} / \kappa_{e}>1$ and therefore strategies to reduce $\kappa_{p}$ are needed to realise the highest possible figure of merit.

KEYWORDS: Molecular thermopower, molecular conductance, density functional theory, single molecule

TEXT: 


\section{Introduction.}

Generation of electricity from heat via the Seebeck effect is silent, environmentally friendly and requires no moving parts. Waste heat from automobile exhausts and industrial manufacturing processes could all be used to generate electricity provided materials with a high thermoelectric figure of merit $Z T$ could be identified. Conversely Peltier cooling using such materials would have applications ranging from on-chip cooling of CMOS-based devices to home refrigerators. Despite several decades of development, the best inorganic thermoelectric materials, such as bismuth telluride (Bi2Te3)-based alloys, possess a figure of merit $Z T$ close to unity only, which is not sufficient to create a viable technology platform for harvesting waste heat. As an alternative, organic thermoelectric materials are now being investigated and are already showing promising values of both $Z T$ and thermopower (Seebeck coefficient) ${ }^{1}$.

The thermopower or Seebeck coefficient $S$ and thermoelectric figure of merit (ZT) of a material or of a nanojunction are defined as

$$
\begin{aligned}
& S=-\frac{\Delta V}{\Delta T} \\
& Z T=-\frac{S^{2} G T}{\kappa}
\end{aligned}
$$

where $\Delta V$ is the voltage difference created between the two ends of the junction when a temperature difference $\Delta T$ is established between them, $G$ is the electrical conductance, $T$ is the ambient temperature and $\kappa$ is the thermal conductance. A key strategy for improving the thermoelectric properties of inorganic materials has been to take advantage of nanostructuring ${ }^{2-4}$, which leads to quantum confinement of electrons and enhanced thermoelectric performance ${ }^{4-10}$. The single-molecule building blocks of organic materials offer the ultimate limit of electronic confinement, with quantised energy level spacings which are orders of magnitude greater than room temperature. Therefore it is natural to examine the thermoelectric performance of single-molecule junctions as a stepping stone towards the design of new materials. The ability to measure thermopower in single-molecule junctions is relatively new ${ }^{11-16}$ and the thermoelectric properties of only a few molecules have been measured. To underpin further exploration and optimisation of single-molecule thermoelectric devices, the aim of this paper is to survey theoretically the thermoelectric performance of a 
family of related single molecules and demonstrate that redox control is a viable strategy for enhancing both $S$ and $Z T$.

Initially, when discussing the figure of merit, we shall focus on the electronic contribution $Z_{e l} T$ to $Z T$, defined by $Z_{e l} T=-S^{2} G T / \kappa_{e l}$, where $\kappa_{e l}$ is the electronic contribution to the thermal conductance. The full figure of merit is then given by ZT $=Z_{e l} T\left(1+\kappa_{\mathrm{p}} / \kappa_{\mathrm{e}}\right)$, where is $\kappa_{\mathrm{p}}$ the phonon contribution to the thermal conductance.Figures 1a,b and Figure 2 show the family of nine molecules investigated, which consist of dithiol-terminated oligo(phenyleneethynylenes) (OPE) containing various central units. Molecules 1 and 2 differ from 3-9, because the latter are conjugated, whereas the anthraquinone central unit of $\mathbf{2}$, which contains pendant oxygens, is cross conjugated, while in $\mathbf{1}$, which is a reduced form of $\mathbf{2}$ with hydrogens attached to the oxygens, conjugation is broken.

When a molecule is weakly coupled to electrodes and the energy $E$ of an electron approaches the energy $\varepsilon_{\mathrm{m}}$ of a molecular orbital, then the transmission coefficient $T(E)$ is approximated by the Breit-Wigner formula $T(E)=4 \Gamma_{1} \Gamma_{2} /\left[\left(E-\varepsilon_{0}\right)^{2}+\left(\Gamma_{1}+\Gamma_{2}\right)^{2}\right]$, where $\Gamma_{1}$ and $\Gamma_{2}$ are the level broadenings due to contact with the left (1) and right (2) electrodes and $\varepsilon_{0}=\varepsilon_{m}-\Sigma_{l}-\Sigma_{2}$ is the resonance energy, obtained by shifting $\varepsilon_{m}$ by the real parts of the corresponding self-energies, $\Sigma_{1}$ and $\Sigma_{2}$. This expression is a consequence of constructive quantum interference and shows that $T(E)$ is a maximum when $E=\varepsilon_{0}$. In contrast, when a pendant group attached to a molecule creates a bound state of energy $\varepsilon$, which interacts with such a molecular orbital, then as shown in ref [18], the above expression is replaced by the modified formula $T(E)=$

$4 \Gamma_{1} \Gamma_{2} /\left[\left(E-\varepsilon^{\prime}\right)^{2}+\left(\Gamma_{1}+\Gamma_{2}\right)^{2}\right]$, where $\varepsilon^{\prime}=\varepsilon_{0}+\alpha^{2} /(E-\varepsilon)$. This expression describes the destructive interference which occurs when an electron resonates with a pendant bound state, because when $E=\varepsilon, \varepsilon^{\prime}$ diverges and $T(E)$ vanishes. It is known ${ }^{17-19}$ that pendant oxygens promote destructive quantum interference and create sharp anti-resonances near the Fermi energy $E_{\mathrm{F}}$, leading to a large suppression in electrical conductance ${ }^{17-23}$. Consequently electrochemical switching ${ }^{24}$ from $\mathbf{1}$ to $\mathbf{2}$ should produce a large decrease in the electrical conductance and it is of interest to determine whether or not $S$ and $Z T$ can also be controlled by varying the oxidation state of $\mathbf{1}$ and $\mathbf{2}$.

\section{Theoretical Methods.}

Transport properties are computed using the ab-initio code SMEAGOL ${ }^{25,26}$ which employs the Hamiltonian provided by the density functional theory (DFT) code SIESTA, ${ }^{27}$ in combination with the nonequilibrium Green`s function formalism. SIESTA uses normconserving pseudo-potentials and linear combinations of pseudo-atomic orbitals to construct the valence states. The calculations used a single zeta (SZ) basis set for the gold leads, which included the $s$ - and $d$-orbitals in the valence to make sure the coupling to the sulphur atoms 
was correctly described. An energy cut-off of 200 Ry was chosen to define the real space grid, necessary to represent the density and the potential and to calculate the Hamiltonian and the overlap matrix elements. The local density approximation (LDA) ${ }^{28}$ was employed to calculate exchange and correlation effects. The molecular coordinates were relaxed in the isolated molecule until the forces were smaller than $0.05 \mathrm{eV} / \AA$, and then included into the "extended molecule" without further relaxation (relaxation of the atomic coordinates inside the extended molecule gave essentially the same results). The "extended molecule" is represented by the dithiolated OPE compound bridged to five atomic gold layers to account for specific properties of the contact region such as adsorption geometry and molecular conformation. Each layer contained 18 atoms, i.e. 6 and 3 atoms along each lattice vector on the (111) surface. One of the sulfurs was bound to a threefold hollow (H) site of the $\mathrm{Au}(111)$ surface ( $\mathrm{Au}-\mathrm{S}$ distance $\sim 0.20 \mathrm{~nm}$ ) and the other one to a gold adatom, which was contacted to the surface in a hollow site ( $\mathrm{Au}-\mathrm{S}$ distance $\sim 0.24 \mathrm{~nm}$ ) following the (111) direction of growth.

Corrected positions of the Fermi energy relative to the HOMO to account for the DFT underestimation of the HOMO-LUMO gap were obtained by applying a scissor-type operator to the bare DFT results (SAiNT ${ }^{29,30}$ ) that moves the occupied and the unoccupied molecular levels downwards and upwards, respectively. This spectral adjustment was chosen to fit the position of such levels as obtained from a combination of experimental UPS, UV/VIS absorption and electrochemical data and has been verified through comparison with electrical measurements on molecules $\mathbf{1 - 9 ^ { 3 1 , 3 2 }}$. The Hamiltonians derived in these arrangements are then used by SMEAGOL to calculate the transmission coefficients $T(E)$.

To calculate thermoelectric quantities ${ }^{17}$ it is useful to introduce the non-normalised probability distribution $P(E)$ defined by

where $T(E)$ is the transmission coefficient for electrons of energy $E$ passing from one electrode to the other, $f(E)$ the Fermi-Dirac function, whose moments are denoted

where $\mathrm{E}_{\mathrm{F}}$ is the Fermi energy. The conductance, $G$ is

where $e$ is the electronic charge, $h$ is the Planck constant, and $T$ is the temperature. The thermopower $S$ is 
the electronic contribution to the thermal conductance $\kappa_{e l}$ is

el $\quad--\quad-$

and the electronic contribution to the figure of merit $Z_{e l} T$ is

All our results were obtained using the above formulae. As an aside, for $E$ close to $E_{F}$, if $T(E)$ varies only slowly with $E$ on the scale of $k_{B} T$ then these expressions take the well-known forms:

where $\quad-\quad$ is the Lorentz number. The latter expression demonstrates the "rule of thumb" that $\mathrm{S}$ in enhanced by increasing the slope of $\ln T(E)$ near $E=E_{F}$.

To compute the phonon contribution $\kappa_{\mathrm{p}}$ to the termal conductance, we evaluate the expression

where is the transmission coefficient for phonons of frequency $\omega$ and is the Boltzmann distribution ${ }^{33}$.

To compute we place molecules $\mathbf{1}$ and $\mathbf{2}$ between simple one-dimensional gold chains and after geometry relaxation using the reaxFF force field within LAMMPS, we extract the dynamical matrix of the system using finite differences to calculate the energy derivatives with respect to $\mathrm{x}, \mathrm{y}$ and $\mathrm{z}$ components of all atoms. The transmission coefficient is then computed by calculating the phonon Green's function connecting the left to the right gold leads, as discussed in ${ }^{34}$.

\section{Results}


Figure 1a shows a dithiol-terminated oligo(phenylene-ethyny-lene) (OPE) containing a dihydroanthracene central unit $\mathbf{1}$ connected via thiol anchor groups to gold electrodes, which mimic a STM tip and a surface. Figure 1c (blue curve) shows that the transmission coefficient $T(E)$ for electrons passing from one electrode to the other is rather featureless in the vicinity of the Fermi energy $\left(E-E_{\mathrm{F}}=0\right)$. Electrochemically oxidising this molecule removes the hydrogens from the - $\mathrm{OH}$ groups to yield the cross-conjugated anthraquinone central unit 2 shown in figure $1 \mathrm{~b}$, which possess pendant oxygens. As expected, figure 1c (green dashed curve) shows that pendant oxygens produce destructive interference in the vicinity of $E_{\mathrm{F}}$, indicated by a sharp decrease in $T(E)$ near $E_{\mathrm{F}}$. Since the electrical conductance $G$ is given by $G=\mathrm{G}_{0} T\left(E_{\mathrm{F}}\right)$, where $\mathrm{G}_{0}$ is the quantum of conductance, one expects that the conductance of 2 to be significantly suppressed. This prediction has been confirmed experimentally only very recently ${ }^{31,32,35,36}$.

For 1 and 2, figure 3 shows the variation of $G, S, \kappa_{e l}$ and $Z_{e l} T$ with temperature and demonstrates that at room temperature, the redox switching from 1 to $\mathbf{2}$ produces a threeorders-of-magnitude suppression of $G$ and $\kappa$ and a large enhancement of $S$ and $Z_{e l} T$. This enhancement is ultimately associated with the appearance of the anti-resonance near $\mathrm{E}_{\mathrm{F}}$, because as noted in equ (8), at low temperatures, $S$ is proportional to the slope of the logarithm of $T(E)$, evaluated at $E=E_{F}$.

To demonstrate that the large room-temperature value of $S=80 \mu \mathrm{V} / \mathrm{K}$ obtained for 2 is unprecedented and much higher than those obtained for other OPE-based molecular wires, figure 4a shows plots of $S$ for the remaining seven members of this family of molecular wires, shown in figure 2. At $300 \mathrm{~K}$, the thermopowers of all other molecules are approximately a factor of two or less than that of $\mathbf{2}$. Literature values for $\mathrm{S}$ obtained experimentally are also significantly lower than that of $\mathbf{2}$. For example, recently-measured values of $\mathrm{S}$ at room temperature include 8.7, 12.9 and $14.2 \mu \mathrm{V} / \mathrm{K}$ for 1,4-benzenedithiol (BDT), 4,4'dibenzenedithiol, and 4,4"-tribenzenedithiol in contact with gold respectively ${ }^{12},-1.3$ to 8.3 $\mu V / K$ for the benzene-based series of benzene-dithiol (BDT), 2,5-dimethyl-1,4benzenedithiol (BDT2Me), 2,3,5,6-tetrachloro-1,4-benzenedithiol (BDT4Cl), 2,3,5,6tetraflouro-1,4-benzenedithiol (BDT4F) and $\mathrm{BDCN}^{11,13}, 7.7$ to $15.9 \mu \mathrm{V} / \mathrm{K}$ for the series BDT, DBDT, TBDT and DMTBDT ${ }^{16},-12.3$ to $13.0 \mu \mathrm{V} / K$ for a series of amine-Au and pyridineAu linked molecules ${ }^{37}$ and -8.9 to $-33.1 \mu \mathrm{V} / K$ for fullerene-based single-molecule junctions ${ }^{15,38,39}$. The thermopower of molecule 2 exceeds all of these values. It also exceeds theoretical predictions of S, provided corrections to DFT are implemented to yield agreement with experiment ${ }^{40,41}$. 
In practice the task of measuring the thermal conductance $\kappa$ is non-trivial and experimental values of $Z T$ are usually not reported in the literature. Figure $4 \mathrm{~b}$ demonstrates that the electronic figure of merit $Z_{e l} T$ of $\mathbf{2}$ is also much higher than that of molecules $\mathbf{1}$ and 3-9 and therefore if the phonon contribution $\kappa_{p}$ to the thermal conductance can be reduced to a value below that of $\kappa_{e}$, this would form the basis of an attractive thermoelectric converter.

To estimate the phonon contribution, we have calculated the phonon transmission coefficients $\tau(\omega)$ for the structures shown in figure 5. These are shown in figure 6 and the resulting temperature dependences for $\kappa_{p}$ obtained by evaluating equation 9 are shown in figure 7 . This shows that for molecule $\mathbf{1}$ and $\mathbf{2}$ attached to gold electrodes, the ratio $\kappa_{p} / \kappa_{e}>>1$ and therefore $Z T \ll Z_{e l} T$. To remedy this suppression, strategies for suppressing the role of parasitic phonons should be implemented. Possible strategies include utilising electrodes made from materials with lower thermal conductance than gold, nanostructuring the electrodes to introduce phonon band gaps, or utilising disordered electrodes which maximise phonon scattering or even introduce phonon localisation ${ }^{42,43,44}$.

\section{Summary}

In summary, we have demonstrated that redox control of single molecules is a viable strategy for enhancing the thermoelectric performance of molecular junctions. Thermopower, unlike electrical conductance, is an intrinsic quantity, which does not scale with the surface area of a monolayer of molecules or with the contact configuration ${ }^{29}$. Therefore the above results for single molecules point the way towards engineering the thermoelectric properties of thin films. The idea of utilising destructive quantum interference to yield enhanced thermoelectric properties was discussed in $^{17}$ and observation that pendant oxygens lead to anti-resonances close to the Fermi energy was highlighted in ${ }^{18,19}$. By employing a corrected DFT Hamiltonian which has been benchmarked against experiments in ref ${ }^{31}$, the above calculations demonstrate that these two effects combine in molecules $\mathbf{1}$ and $\mathbf{2}$ to yield enhanced electrochemically-switchable values of $S$ and $Z_{e l} T$, which are competitive with the best available organic materials. Such values of $S$ and $Z_{e l} T$ are highly unusual, as demonstrated by comparison with the family of related molecules 3-8 and comparison with recent experiments. For the future, it will be of interest to examine the thermoelectric properties of related families of molecules with pendant oxygens, such as the fluorene-based example of ref $^{19}$. 


\section{Figures}

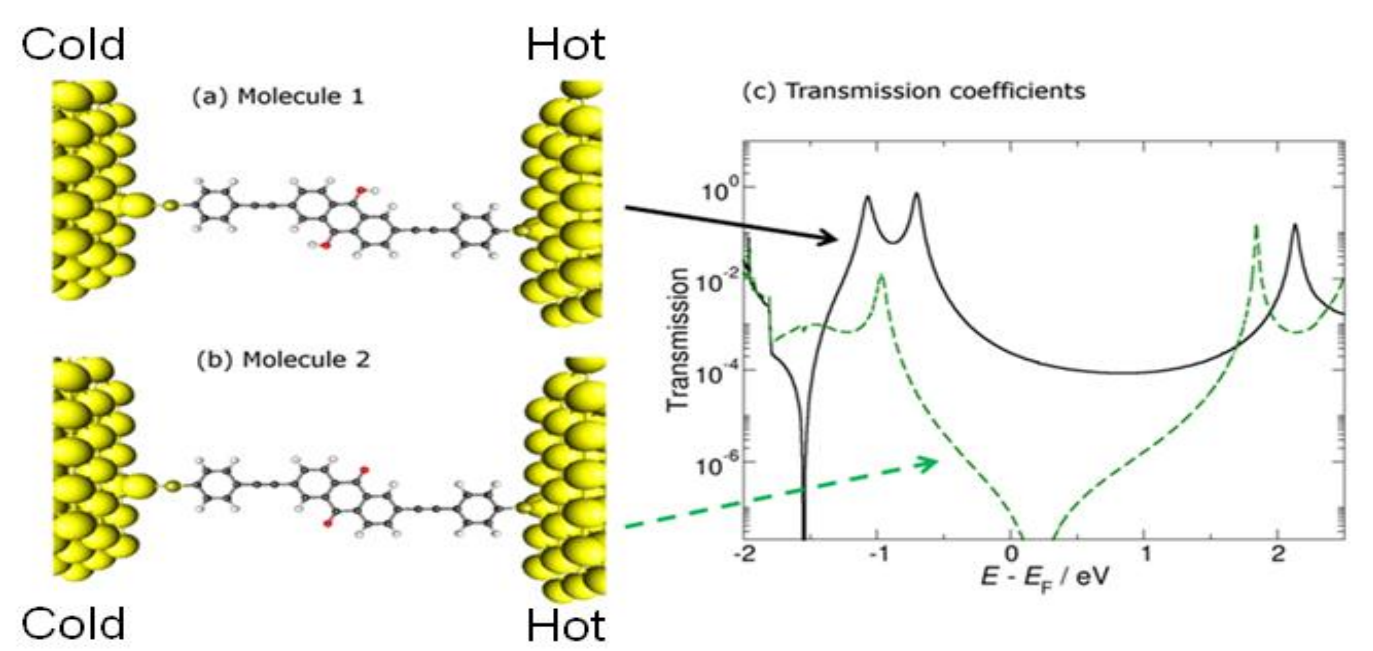

Figure 1. (1a) A dithiol-terminated oligo(phenylene-ethyny-lene) (OPE) containing a dihydroanthracene central unit 1 connected via thiol anchor groups to gold electrodes. (1b) The cross-conjugated anthraquinone central unit 2. (1c) Transmission coefficients $T(E)$ for $\mathbf{1}$ (continuous curve) and $\mathbf{2}$ (dashed curve).

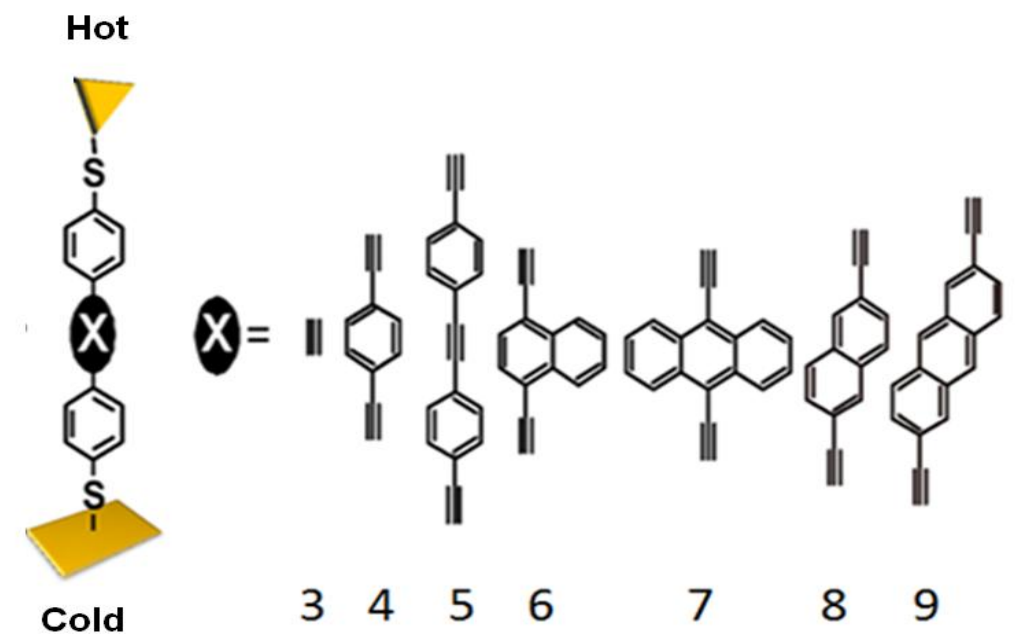

Figure 2. Seven OPE-based wires with different lengths and central units, labeled 3-9. 

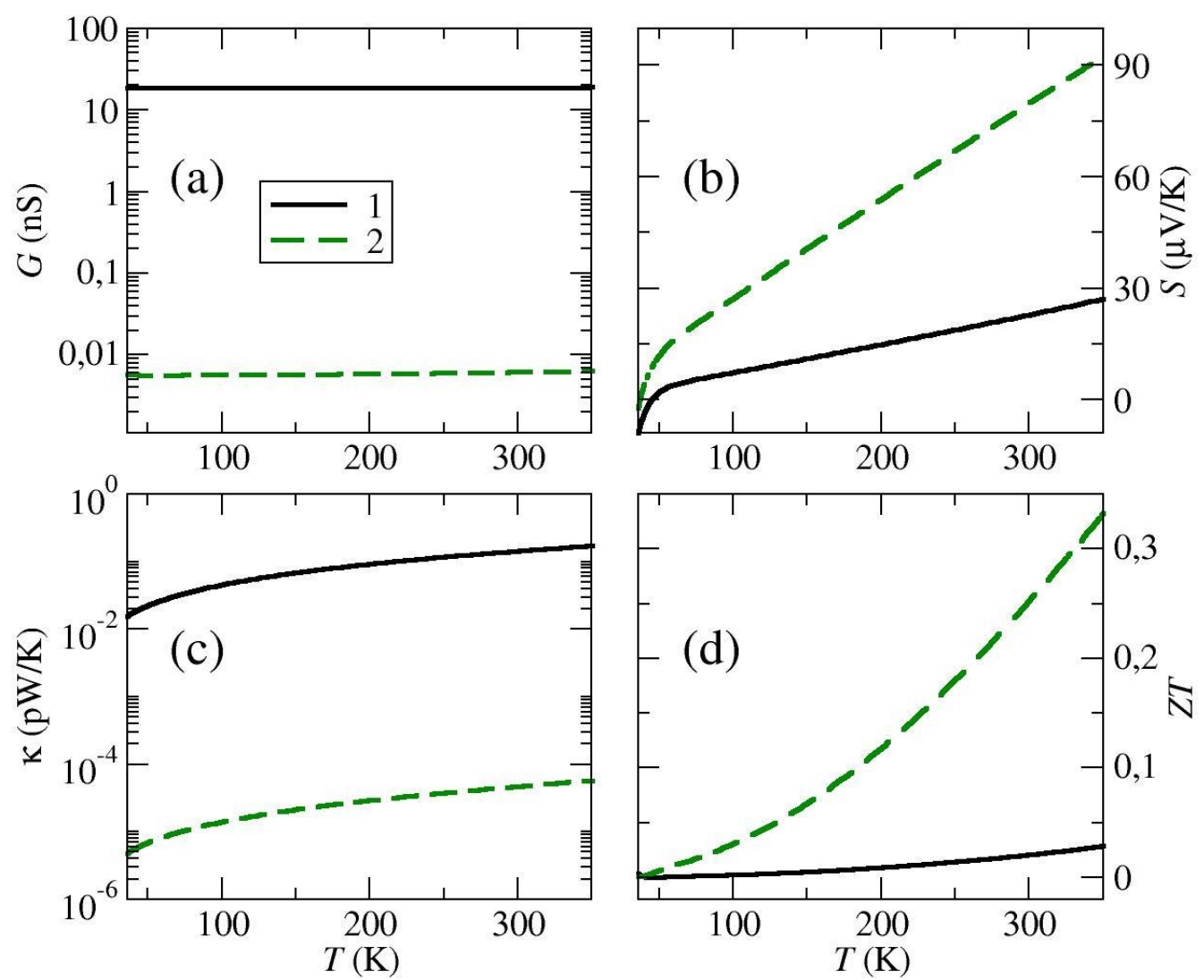

Figure 3. (2a), (2b), (2c) and (2d) show, respectively, the variation of the electrical conductance $(G)$, the Seebek coefficient $(S)$, the thermal conductance $(\kappa)$ and the figure of merit $(Z T)$ with temperature for molecules $\mathbf{1}$ and $\mathbf{2}$.
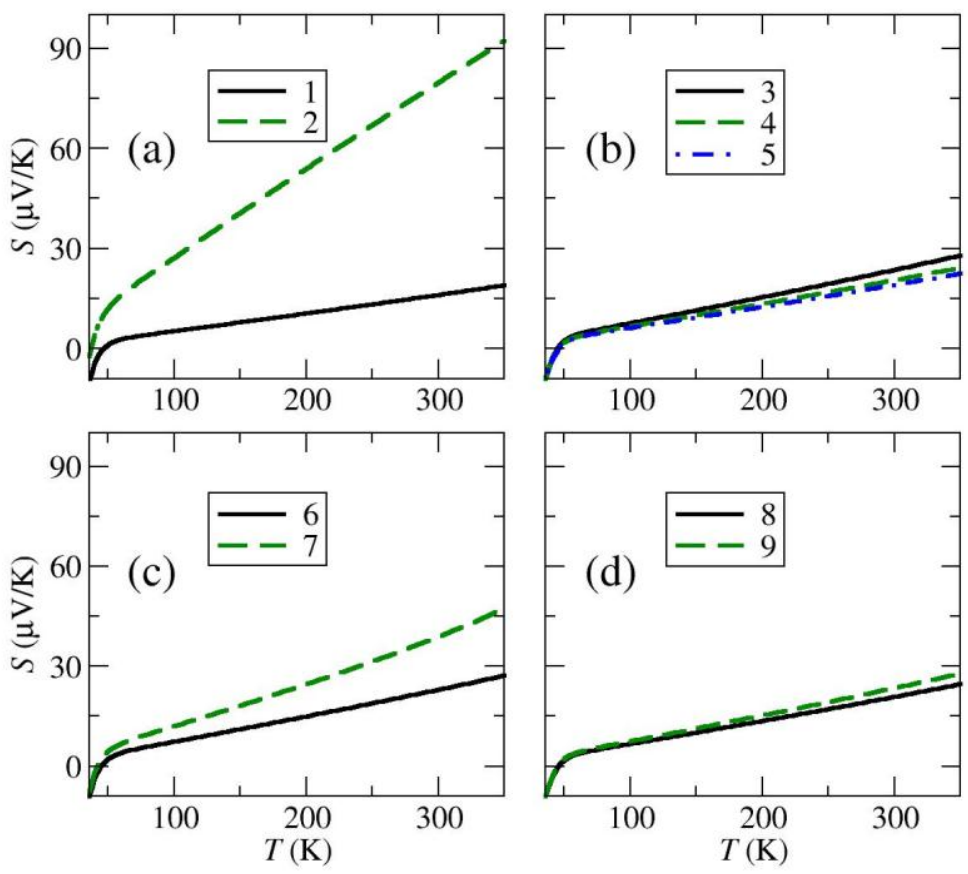


\section{Figure 4a}
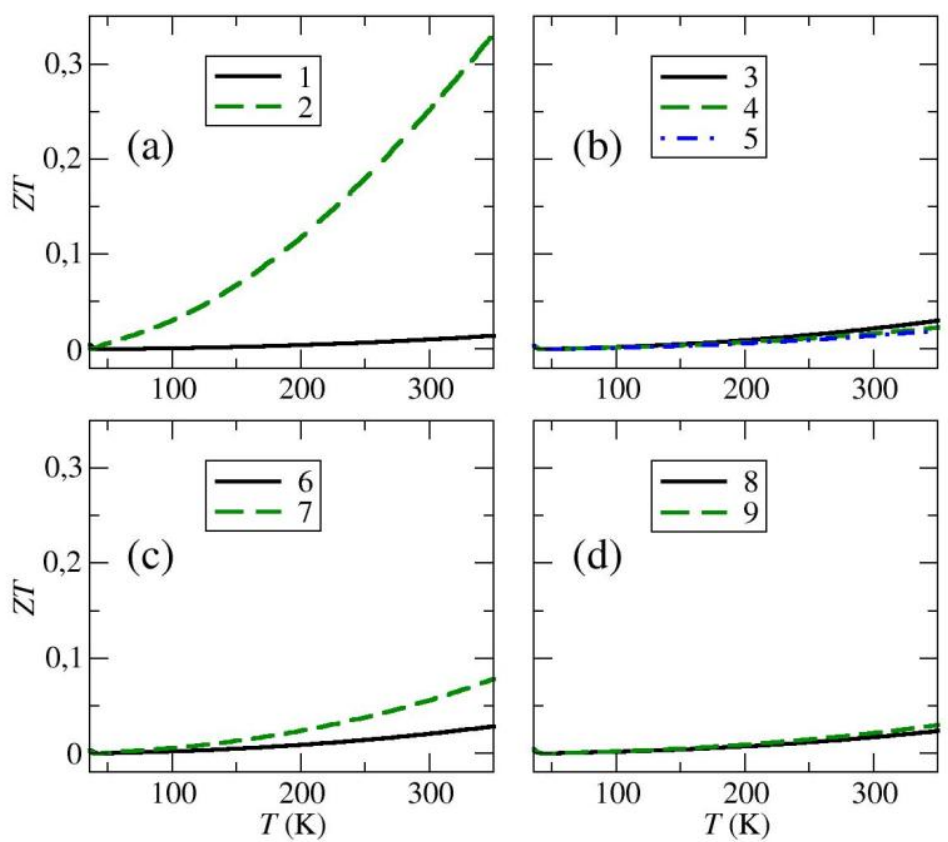

Figure 4b

Figure 4. (4a) Variation of $S$ with temperature for the nine OPE-based wires of Figs. 1 and 3. (4b) Variation of $Z T$ with temperature for the nine OPE-based wires of Figs 1 and 3.

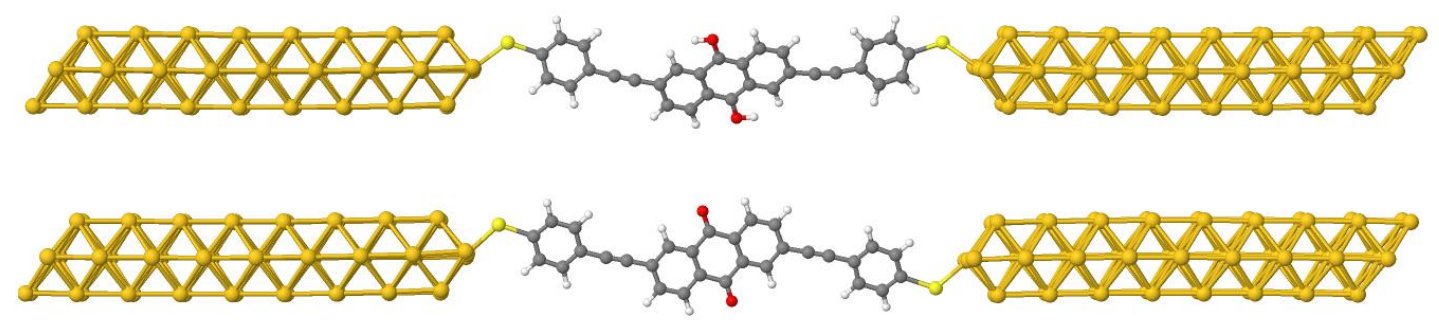

Figure 5. Optimized geometries of molecules $\mathbf{1}$ and $\mathbf{2}$ connected to simple gold chains. 


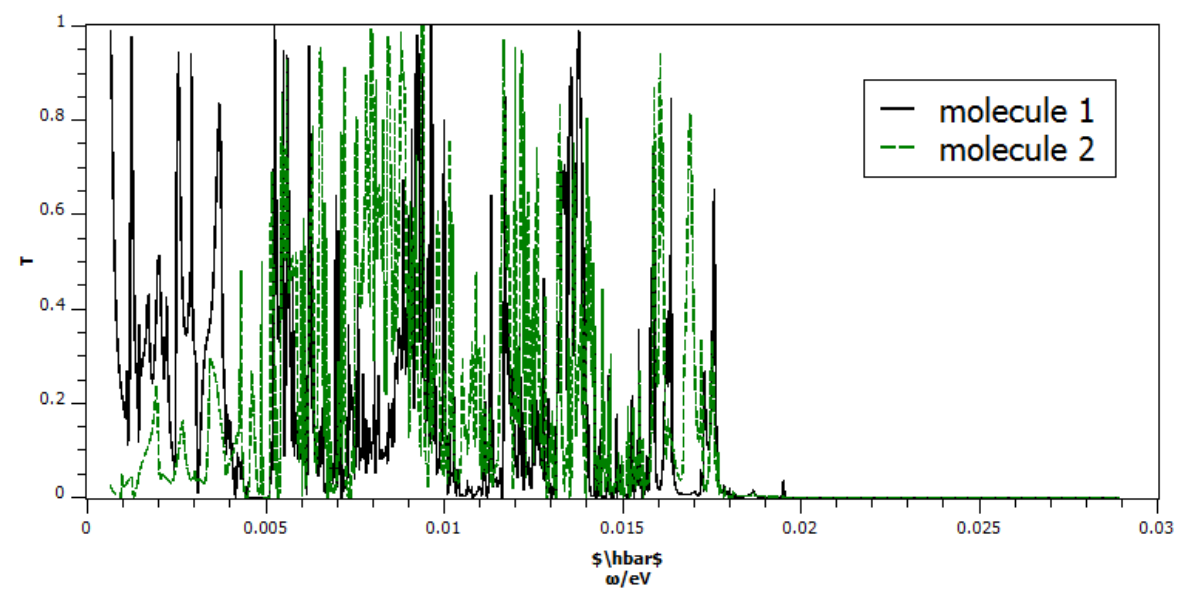

Figure 6. The phonon transmission coefficients for the molecular systems 1 (black) and 2 (green) shown in figure 5

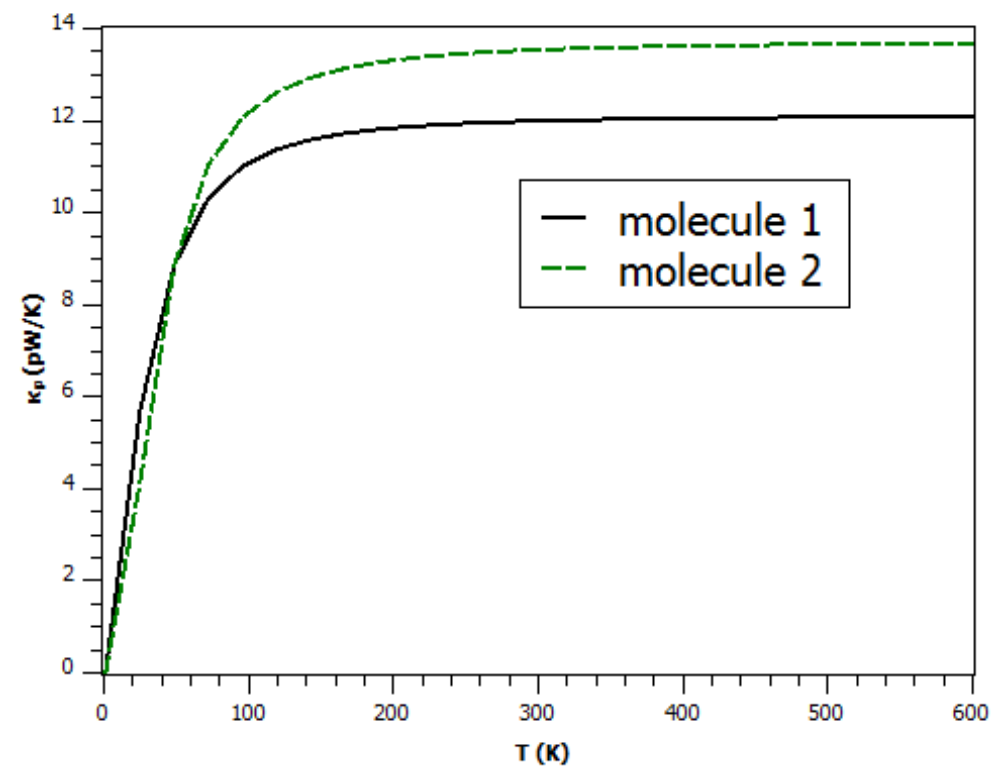

Figure 7. Thermal conductance for the molecular systems shown in Figure 5.

AUTHOR INFORMATION

\section{Corresponding Author}

*E-mail c.lambert@lancaster.ac.uk.

\section{Author Contributions}

The authors contributed equally to this work. 


\section{References}

[1] O. Bubnova, Z. U. Khan, A. Malti, S. Braun, M. Fahlman, M. Berggren and X. Crispin, Nature Materials, 10, 429 (2011)

[2] R. Venkatasubramanian, E. Siivola, T. Colpitts, and B. O’Quinn, Nature 413, 597 (2001).

[3] T. C. Harman, P. J. Taylor, M. P. Walsh, and B. E. LaForge, Science 297, 2229 (2002).

[4] B. Poudel, Q. Hao, Y. Ma, Y. Lan, A. Minnich, B. Yu, X. Yan, D. Wang, A. Muto, D. Vashaee, X. Chen, J. Liu, M. S. Dresselhaus, G. Chen, and Z. Ren, Science 320, 634 (2008).

[5] K. F. Hsu, S. Loo, F. Guo, W. Chen, J. S. Dyck, C. Uher, T. Hogan, E. K. Polychroniadis, and M. G. Kanatzidis, Science 303, 818 (2004).

[6] M. S. Dresselhaus, G. Chen, M. Y. Tang, R. G. Yang, H. Lee, D. Z.Wang, Z. F. Ren, J. P. Fleurial, and P. Gogna, Mater. Res. Soc. Symp. Proc. 886, 170056 (2005).

[7] T. M. Tritt, B. Zhang, N. Gothard, J. He, X. Ji, D. Thompson, and J. W. Kolis, Mater. Res. Soc. Symp. Proc. 886, 170104 (2005).

[8] J. Martin, G. S. Nolas, W. Zhang, and L. Chen, Appl. Phys. Lett. 90, 222122 (2007).

[9] P. N. Alboni, X. Ji, J. He, N. Gothard, and T. M. Tritt, J. Appl. Phys. 103, 113707 (2008).

[10] N. Gothard, X. Ji, J. He, and T. M. Tritt, J. Appl. Phys. 103, 054314 (2008).

[11] Malen, J.A., S.K. Yee, A. Majumdar, R.A. Segalman, , Chem. Phys. Lett. 491, 109 (2010).

[12] P. Reddy, S.-Y. Jang, R. A. Segalman, and A. Majumdar, , Science 315, 1568 (2007).

[13] K. Baheti, J. A. Malen, P. Doak, P. Reddy, S. Y. Jang, T. D. Tilley, A. Majumdar, and R. A. Segalman, , Nano Lett. 8, 715 (2008).

[14] S. K. Yee, J. A. Malen, A. Majumdar, and R. Segalman, , Nano Lett. 11, 4089 (2011).

[15] S. Yee, J. Malen, P. Reddy, R. Segalman, and A. Majumdar, Proc. of the 14th Int. Heat Transfer Conf. IHTC14, Washington, DC, USA, 2010.

[16] J. A. Malen, P. Doak, K. Baheti, T. D. Tilley, A. Majumdar, and R. A. Segalman, Nano Lett. 9, 3406 (2009).

[17] C. M. Finch, V. M. García-Suárez, and C. J. Lambert, Phys. Rev. B 79, 033405 (2009).

[18] T. A. Papadopoulos, I.M. Grace and C.J. Lambert, , Phys. Rev. B74 193306 (2006).

[19] C. Wang, M. R. Bryce, J. Gigon, G. J. Ashwell, I. Grace, and Colin J. Lambert, , J. Org. Chem. 73, 4810 (2008).

[20] J. P. Bergfield, M. Solis, C. A.,Stafford, , ACS Nano 45314 (2010).

[21] A. B. Ricks, G. C. Solomon, M. T. Colvin, A. M. Scott, K. Chen, M. A. Ratner, and M. R. Wasielewski, J. Am. Chem. Soc. 132, 15427 (2010). 
[22] G. C. Solomon, J. P. Bergfield, C. A. Stafford, and M. A. Ratner, Beilstein J. Nanotechnol. 2, 862 (2011).

[23] M. Buerkle, L. A. Zotti, J. K. Viljas, D. Vonlanthen, A. Mishchenko, T. Wandlowski, M. Mayor, G. Schoen, and F. Pauly, , Phys. Rev. B 86115304 (2012).

[24] T. Markussen, J. Schiötz, and K. S. Thygesen, J. Chem. Phys. 132, 224104 (2010)

[25] A. R. Rocha, V. M. Garcia-Suarez, S. W. Bailey, C. J. Lambert, J. Ferrer, and S. Sanvito, Phys. Rev. B 73, 085414 (2006).

[26] A. R. Rocha, V. M. Garcia-Suarez, S. W. Bailey, C. J. Lambert, J. Ferrer, and S. Sanvito, Nat .Mater 4, 335 (2005).

[27] J. M. Soler, et al., J. Phys. Condens. Matter 14, 2745 (2002).

[28] J. P. Perdew and A. Zunger, Phys. Rev. B 23, 5048 (1981).

[29] D. J. Mowbray, G. Jones, and K. S. Thygesen, J. Chem. Phys. 128, 111103 (2008).

[30] V. M. García-Suárez and C. J. Lambert, New Journal of Physics 13, 053026 (2011).

[31] V. Kaliginedi, P. Moreno-Garcia, H. Valkenier, W. J. Hong, V. M. García-Suárez, P. Buiter, J. L. H. Otten, J. C. Hummelen, C. J. Lambert, and T. Wandlowski, J. Am. Chem. Soc. 134, 5262 (2012)

[32] W. J. Hong, H. Valkenier, G. Meszaros, D. Z. Manrique, A. Mishchenko, A. Putz, P. M. García, C. J. Lambert, J. C. Hummellen, and T. Wandlowski, Beilstein J. Nanotech. 2, 699 (2011).

[33] L.G.C. Rego and G. Kirczenow, Phys. Rev. Lett. 81232 (1998)]

[34] D. Nozaki, H. Sevincli, W. Li, R. Gutierrez, and G. Cuniberti, Phys. Rev. B 81, 235406 (2010)].

[35] C. M. Guedon, H. Valkenier, T.Markussen, K. S. Thygesen, J. C. Hummelen and S. J. van der Molen, Nature Nano. 7, 304 (2012).

[36] S. Ballmann, R. Härtle, P. B. Coto, M. Elbing, M. Mayor, M. R. Bryce, M. Thoss and H. B. Weber, Phys. Rev. Lett.. 109, 056801 (2012).

[37] Widawsky, J.R.; Darancet, P.; Neaton, J.B.; Venkataraman L., Nano Lett., 12, 354-358 (2012)

[38] Yee, S.K.; Malen, J.A.; Majumdar, A.; Segalman, R., Nano Lett., 11, 4089 (2011).

[39] Evangeli, C., Gillemot, G., Leary E., González M. T., Rubio-Bollinger G., Lambert C. J., Agraït N., Nano. Lett. 132141 (2013).

[40] S. Y. Quek, H. J. Choi, S. G. Louie, and J. B. Neaton, ACS Nano 5, 551 (2010)

[41] M. Bürkle, L. A. Zotti, J. K. Viljas, D. Vonlanthen, A. Mischenko, T. Wandlowski, M. Mayer, G. Schön, and F. Pauly, Phys. Rev. B 86, 115394 (2012)

[42]G. Fagas, A.G. Kozorezov, et al. Phys. Rev. B 606459 (1999)

[43]A. Kambili, G. Fagas, V.I. Fal'ko, VI and C.J. Lambert, Phys. Rev. B 6015593 (1999)

[44]C.J. Lambert, J. Low Temp. Phys. 59123 (1985) 
NOTES

The authors declare no competing financial interest.

\section{ACKNOWLEDGEMENTS}

This work was supported by the European Union (FP7) ITN NANOCTM and by the EPSRC. VMGS thanks the Spanish Ministerio de Economía y Competitividad for a Ramón y Cajal fellowship (RYC-2010-06053). 